\title{
Design of a College English Mobile Learning System Based on CAD Model
}

\author{
https://doi.org/10.3991/ijet.v13i04.8477 \\ Xin Zhang \\ Dalian Polytechnic University, Dalian, China \\ Jingxuan $\left.\mathrm{Bi}^{(}\right)$ \\ Dalian Medical University, Dalian, China \\ jingxuanbi@126.com
}

\begin{abstract}
With the development of mobile Internet technology and the advent of the information age, people's demand for mobile learning is growing and the studies on mobile English learning platform are also drawing more and more attention. In this article, firstly, the background of mobile learning is introduced and a Conversation-Activity-Distribution theoretical model, CAD, is proposed with activity theory, situation cognition theory and distributed cognition theory as the cognitive basis. Then, the students' demand and expectations for implementation, function and content of the mobile learning system are learned by the combination of experiential researches and questionnaire surveys. A model of college English mobile learning system is proposed based on the CAD model, the overall structure of the model is discussed and its various functional modules introduced. With vocabulary learning as case design, the process design, function design and adaptive design of the system are introduced and the functional interface to the system demonstrated. Theoretically, this article enriches the theoretical basis of mobile learning and improves the methods of mobile learning system. Practically, through the studies on related learning theories, it puts forward a CAD theoretical model and carries out the case study on English vocabulary, which are conducive to the popularization of mobile learning in English learning and the improvement of teaching quality.
\end{abstract}

Keywords-Mobile learning; CAD; System model; System requirements; English vocabulary

\section{Introduction}

Facing with the opportunities and challenges posed by globalization, the core competitiveness of a country has become more focused on the cultivation of qualified personnel. In the process of internationalization, the requirements for the English proficiency of qualified personnel have also been gradually raised [1]. As a part of higher education, English helps to introduce Chinese traditional culture and advanced technology to the world, as well as to exchange with other countries in friendship, economy and trade, politics and so on [2]. The "College English Curriculum Re- 
quirements" issued by the Ministry of Education clearly states that college students' ability to apply English in study, work and social interaction should be improved so as to meet the needs of social development and international exchanges [3]. It is the best way to increase the chances for English learning and improve the efficiency of English learning by applying the computer information technology to meet the needs of students for learning anytime and anywhere [4]. The theory of mobile learning is born and getting mature with this idea. At present, the computer network and mobile communication industry is becoming more and more mature, and many scholars have carried out the studies on the concept, essence, standardization and other hot topics of mobile learning [5].

As of December 2016, the number of China's mobile smart phone users has increased by more than 12 million, reaching to 1.304 billion, of which more than half were $4 \mathrm{G}$ users. At present, there is more than one smart phone per person in China [6]. It indicates that the mobile learning hardware and mobile network can completely meet the demand. Related studies with focus on the design of mobile learning system in university are carried out in this article. Firstly, the connotation and the related research background of mobile learning are summarized and refined. A ConversationActivity-Distribution theoretical model, CAD, is proposed based on conversation theory, activity theory and distribution cognitive theory [7]. Through the combination of experiential researches and questionnaire surveys, this article studies the status quo of college students' mobile learning, their functional and content requirements, personalized needs, etc. On this basis, the functional model of the system is designed based on the CAD model. Finally, the system design is completed and the system interface is displayed and explained with English vocabulary as the actual case [8]. The research of this article provides a new theoretical framework for the design of college English mobile learning system, which meets the needs of mobile learning for college students.

\section{Research background of mobile learning}

\subsection{Analysis of domestic research status}

In 2004, Dr. Ye Chenglin made an in-depth analysis on the concept, research status and key issues of mobile learning in his essay, A Review of Mobile Learning Research. Mobile learning enables learners, as the main body of learning, to arrange related learning according to their own schedule, anytime and anywhere, which is a reversal of traditional education mode [9]. According to the literature survey and statistics, the current research classification of mobile learning in China is mainly as shown in Table 1.

At present, domestic researches on mobile learning mainly focuses on the theoretical part, without innovations. There are some cases in practical application, but they mainly concentrate on summarizing and refining the rules and experience of mobile learning. This article aims to expand the application research and broaden the research scope of mobile learning (Shen et al., 2009). 
Table 1. The mobile learning research classification

\begin{tabular}{|l|l|}
\hline \multicolumn{1}{|c|}{ Classification } & \multicolumn{1}{c|}{ Content } \\
\hline Theory & Theoretical basis, learning model, curriculum design, condition analysis \\
\hline Demonstration & Case analysis, living example, Survey \\
\hline Technology & $\begin{array}{l}\text { Implementation model, Mobile Terminal, Development technology, Resource } \\
\text { design, Key technology }\end{array}$ \\
Group & Basic education, Higher education, Adult education \\
\hline Field & Language study, Enterprise training, Subject teaching \\
\hline
\end{tabular}

\subsection{Analysis of research status abroad}

The number of foreign literature on mobile learning was retrieved in the CNKI, and 415 records were obtained by searching keywords "mobile learning" and "mlearning". The distribution dates and the number of annual publications of the foreign literature are as shown in Figure 1.

\section{document}

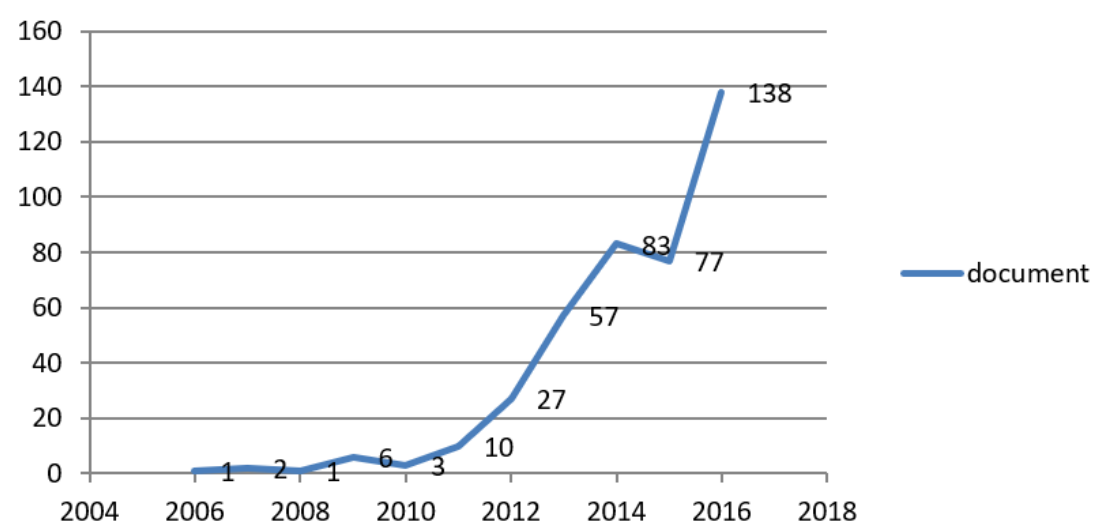

Fig. 1. The document year distribution diagram

It can be seen from the figure that the amount of research literature on mobile learning is basically increasing year by year with a high rate [10]. The content of mobile learning researches abroad are more focused on mobile devices, and their qualities are much better than that of the domestic researches.

\subsection{Introduction of related technologies}

Just as the research of mobile learning abroad concentrates on mobile devices, the mobile learning support technology is the key to implementing English mobile learning system. 
Terminal operating system layer: Mobile devices mainly run through the embedded operating system modes, including four major types at present, Microsoft, Android, Google and Apple, among which the Android and Apple operating systems are the mainstream in the market. Each of these two-mobile device operating systems has its advantages and disadvantages.

Implementation layer: This layer mainly includes five aspects, SMS which realizes learning and communication via short messages; MMS which realizes learning and communication via images, sound, animation and other multimedia information with MMS as the carrier; link browsing which realizes learning and communication by access to the Internet through the Wifi, 3G, 4G modes; storing and carrying which copies learning materials using mobile devices such as USB; and the software which realizes learning and communication mainly by installing APP to mobile devices.

Wireless communication layer: Mobile learning mainly includes two types, networking and non-networking. The network is the vital force of mobile learning. At present, the network that the wireless technology uses mainly include the infinite network, WiFi, and the $3 \mathrm{G}$ and $4 \mathrm{G}$ network provided by telecommunication companies. The next generation of mobile network, $5 \mathrm{G}$, is also in the research phase. The continuous innovation of these wireless technologies has enhanced the convenience and speed of mobile learning.

Mobile learning is a highly personalized individual way of learning. In this article, mobile devices and network related technologies are used for research so as to build a college English mobile learning system platform.

\section{Studies on the theoretical models of mobile learning}

\subsection{Activity theory, situation cognition theory, and distributed cognition theory}

Activity theory. The unity of consciousness and activity is the most basic hypothesis of activity theory. Activity system mainly consists of six elements: subject, tool, and object, division of labor, community and rule. The activity system aims to achieve the transformation from object to result. Some scholars have expanded the activity theory to form a mobile learning framework with two layers, technology and media [11].

Situation cognition theory. Situated cognition emphasizes the social network and activity system where the real behavior takes place. The single learning environment and the internal psychology of learners cannot explain the mechanism of learning. Thus, it is necessary to understand the mobile learning on the basis of the coordination and interaction between people and situations. Situation cognition theory holds that learning is the activity of self-construction by interacting with the learning environment, from which the importance of the external learning environment can be seen. It emphasizes the importance of external learning environment for learning [12].

Distributed cognition theory. Distributed cognition theory holds that mastering learning resources must be decentralized and it should be achieved through the inter- 
actions between individuals and between individuals and learning materials. Distributed cognitive scholars believe that cognitive distribution includes intra individual, inter individual, media, environment, culture, society, time and so on. There are three distinguished features of distributed cognition. Communication is a prerequisite; shared information is aggregated information; and the elements of distributed systems need to depend on each other to achieve tasks [13]. In mobile learning system, the device can be carried around, which can improve the self-organization of the system, so that the self-cognition is improved.

\subsection{CAD: Conversation-Activity-Distribution Model}

Structure of CAD Model. Based on activity theory, situation cognition theory, and distributed cognition theory, the structure of the CAD model is a rotated octahedron as shown in Figure 2.

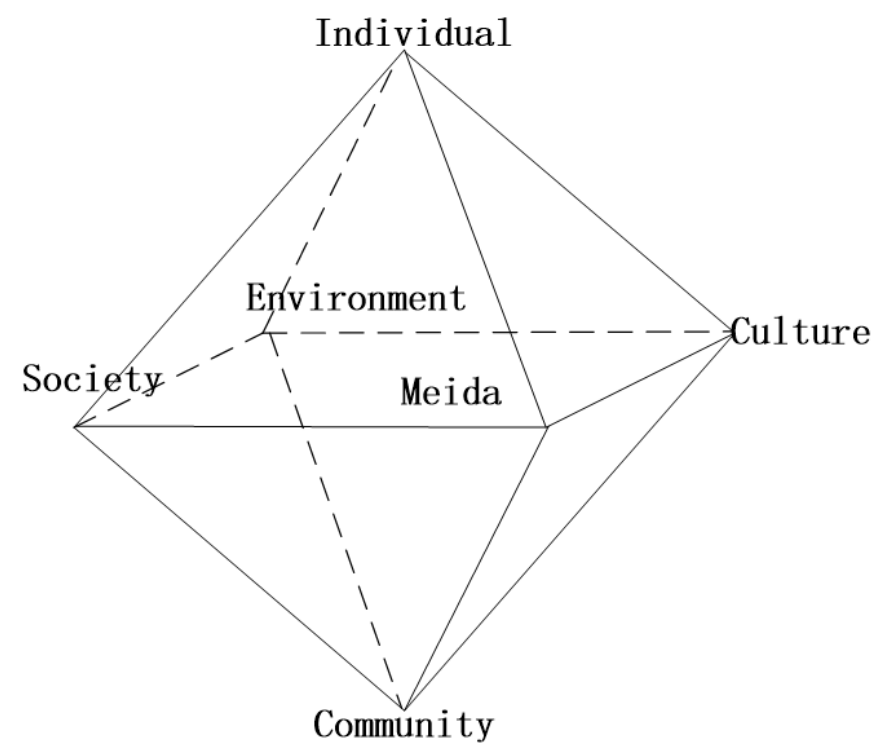

Fig. 2. The structure of CAD model

In Figure 2, the upper vertex represents learners, and the lower one refers to communities. The four vertices of the middle plane represent the media, the environment, the culture and the society from the distributed cognitive theory respectively. This layer is referred to as the connection layer, which is used for the cognitive distribution and the construction of the meaning of learning.

CAD applies conversation theory, activity theory and distributed cognition theory, which provide support for CAD from different perspectives. Activity theory provides a guarantee for the effectiveness of CAD system design, and the distributed theory extends the scope of knowledge representation and storage. 
CAD and model analysis. Compared to other models, CAD has its particularity in specific aspect. CAD is divided into the following four subsystems based on activity theory. (1) Production subsystem can achieve seamless connection with real life as mobile learning has a good randomness. Taking animal related English learning as an example, students can learn while visiting the zoo or animal information can be transferred to the mobile device in the form of pictures through the mobile learning platform, so that the vocabulary of the animals can be more easily remembered by the students. (2) Consumption subsystem helps learners to locate the current activities and be consistent with the overall learning community, as the mobile platform is a community for all learning bodies. (3) The distribution subsystem and communication subsystem use social rules and constraints to enable the subsystems to operate based on the rules of the activity theory [14].

CAD and teaching interaction. The key words of the learner's main learning behavior are control, situation and communication, which correspond to the humancomputer interaction, the physical environment and the means of communication respectively, which achieve their goal through "conversation". Transformation analysis of motivation-activity, goal-behavior, and condition-to-action is needed in order to achieve better learning outcomes.

\section{Analysis of system requirements and introduction of functional model}

\subsection{Analysis of system requirements}

Research methods and objects. Combining experiential research and questionnaire survey, a survey was conducted on freshmen and sophomores in a normal university in China. With stratified sampling method, 21 freshmen and 22 sophomores were sampled, and 40 valid questionnaires and interviews were conducted. The sax ration of the samples was 1:1, with 20 effective samples for each gender.

\section{Research results and analysis.}

Application status analysis. For the interviewees that conducted mobile learning, the survey shows that $45 \%$ of them used laptops, $15 \%$ desktops, $38 \%$ mobile phones and $2 \%$ other mobiles. Moreover, the number of mobile phone users for mobile learning is increasing year by year. More than $70 \%$ of the respondents hoped to receive training for mobile learning and wished to maintain the continuity of learning through mobile learning.

At present, the mobile learning that the respondents receive are mainly implemented by downloading files to local machine, interactive learning through APP, access to internet by mobile, mobile newspapers and so on. As to the implementation of mobile learning, respondents are concerned about the terminal configuration requirements, fluency of course learning, network and communication costs, richness of resources, learning initiative motivation and other aspects [15].

Requirements of mobile learning courses. Mobile learning is mainly a type of fragmented learning in spare time. Its duration requirements are as shown in Table 2. 
Paper-Design of a College English Mobile Learning System Based on CAD Model

Table 2. The lasting time of mobile learning

\begin{tabular}{ccccc}
\hline Less than 5 minutes & $\mathbf{5 - 1 0}$ minutes & $\mathbf{1 0 - 1 5}$ minutes & Above 15 minutes & Total \\
\hline $20 \%$ & $42.5 \%$ & $32.5 \%$ & $5 \%$ & $100 \%$ \\
\hline
\end{tabular}

It can be seen from the table that most of the expected time for mobile learning is concentrated in 5-10 minutes and 10-15 minutes, which is in line with memory science, as the short time learning of 5-15 minutes is more focused and less easy to be disturbed.

The demand for the presentation model of mobile learning is as shown in Table 3.

Table 3. The demand for the presentation model of mobile learning

\begin{tabular}{ccccccc}
\hline Voice frequency & Video & Text & Game & Animation & Figure & Other \\
\hline $67.5 \%$ & $55 \%$ & $52.5 \%$ & $47.5 \%$ & $30 \%$ & $30 \%$ & $0 \%$ \\
\hline
\end{tabular}

The reason that audio and video become the most popular learning media is mainly due to their sensory effects. They are more flexible and more vivid, and they can be more fluently played on the phone than on the internet.

Functional requirements of mobile learning platform. Query function. It should help learners to connect the learning content, sort out knowledge, and consolidate review. Learning function. It should enable respondents to use mobile phones to study at any time and any place, and use mobile learning for review before and after class. Exercising function. Question types should include blank fill questions, multiple choice questions, connection questions, and game questions. Score recording function. Score query should help respondents to know their own learning process and dynamic changes. Learning reminding function. It should remind learners to learn on time that they set as free time for themselves.

\subsection{Introduction of system function models}

The design of College English mobile learning system follows integrity, practicability, modularity, adaptability, intelligence and other principles. The overall function design has three roles: student, teacher and administrator. As the center of the system, students take the initiative to acquire knowledge, test himself or learn through collaboration. Teachers play the role of supervision and guidance. Administrators are responsible for system maintenance and management of students' and teachers' information.

As shown in Figure 3, the functional structure of the system includes creation module, personalized learning module, evaluation and feedback module and management module. Mobile learning services are conducted in the foreground and data resource management is conducted in the background. 


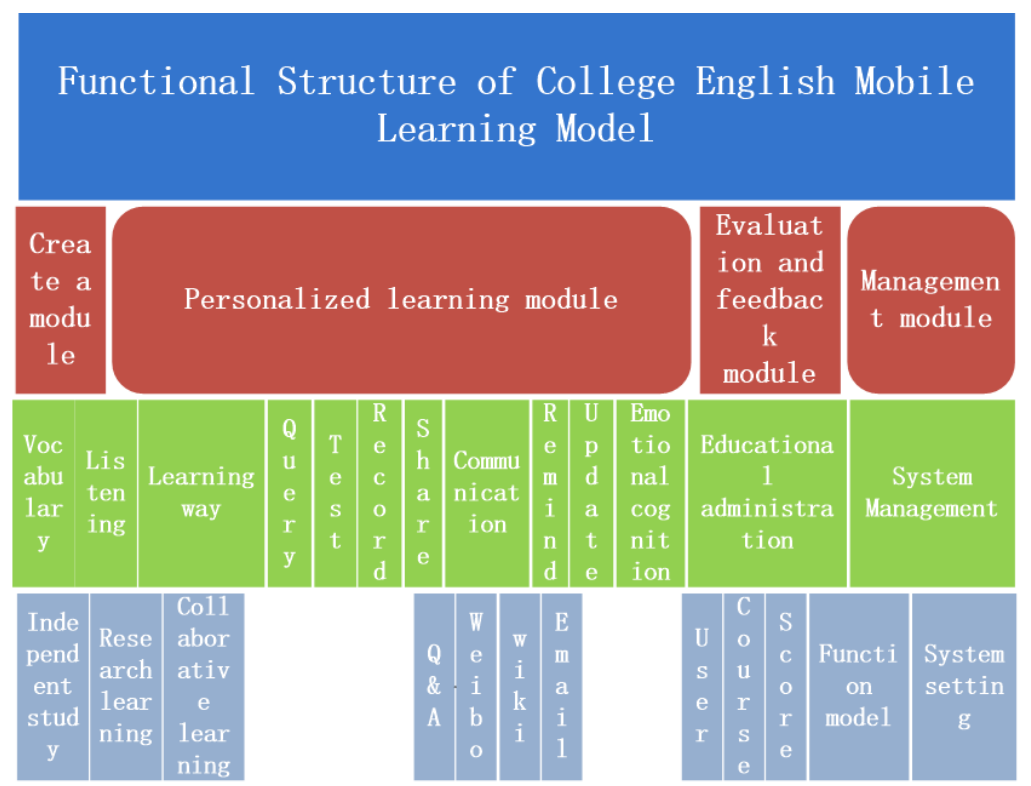

Fig. 3. The system function diagram

The functional modules of the system consist of creation module, personalized learning module, evaluation and feedback module and management module. Personalized learning module is the core of the system, including learning style, inquiry, assessment, recording, sharing, communication, reminding, updating, learning, emotional perception and other functions.

\section{Design of college students' English vocabulary mobile learning system}

In view of the process design, function design and adaptive design process of the system mentioned in the previous chapters, the mobile learning system is introduced based on CAD model with vocabulary learning as a case study in this chapter. In addition, the functional interface to the system is demonstrated. The flow chart of the vocabulary learning system is as shown in Figure 4.

Authentication, learning goal setting, learning level measurement, learning plan generation, learning activity and exercise activity constitute the main process of the system. Vocabulary retrieval, starting point of learning and learning plan adjustment 


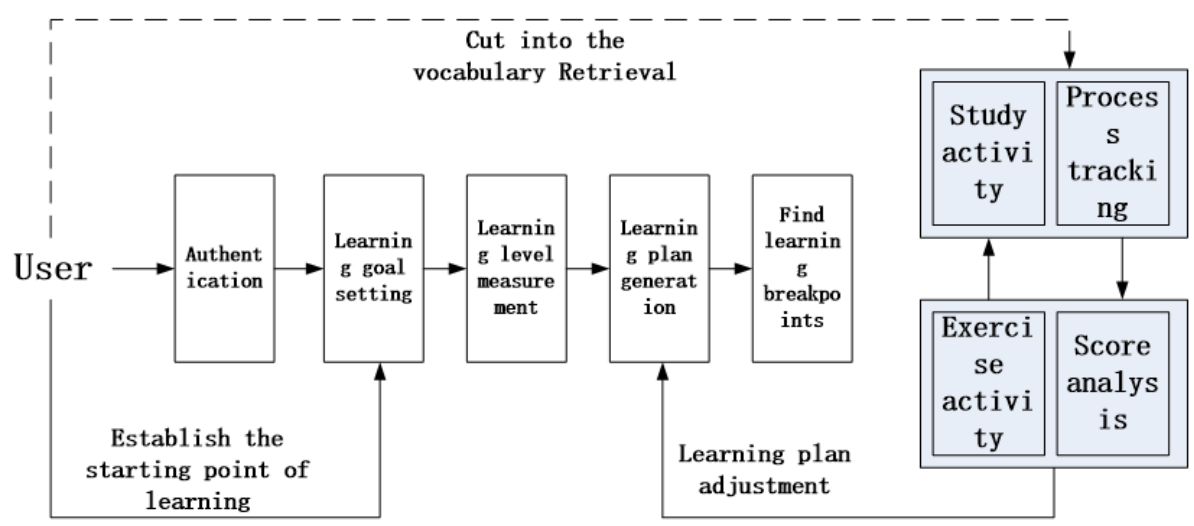

Fig. 4. The flow chart of the vocabulary learning system

Figure 5 is the screenshot of the test session of the personalized learning module of English vocabulary mobile learning system. For this interface, the user can directly select different modules in the menu bar on the left, and then return to the main interface by clicking the button in the middle of the interface.

Compared to the test interface, vocabulary system practice mode is to help students improve their vocabulary. As shown in Figure 6, in the practice mode, the system analyzes different options and interprets the key words in relation to the same multiple choice question.

Other functions will not be described through screen shots here. The English vocabulary mobile learning system based on the CAD theoretical model can help learners to improve their English proficiency.

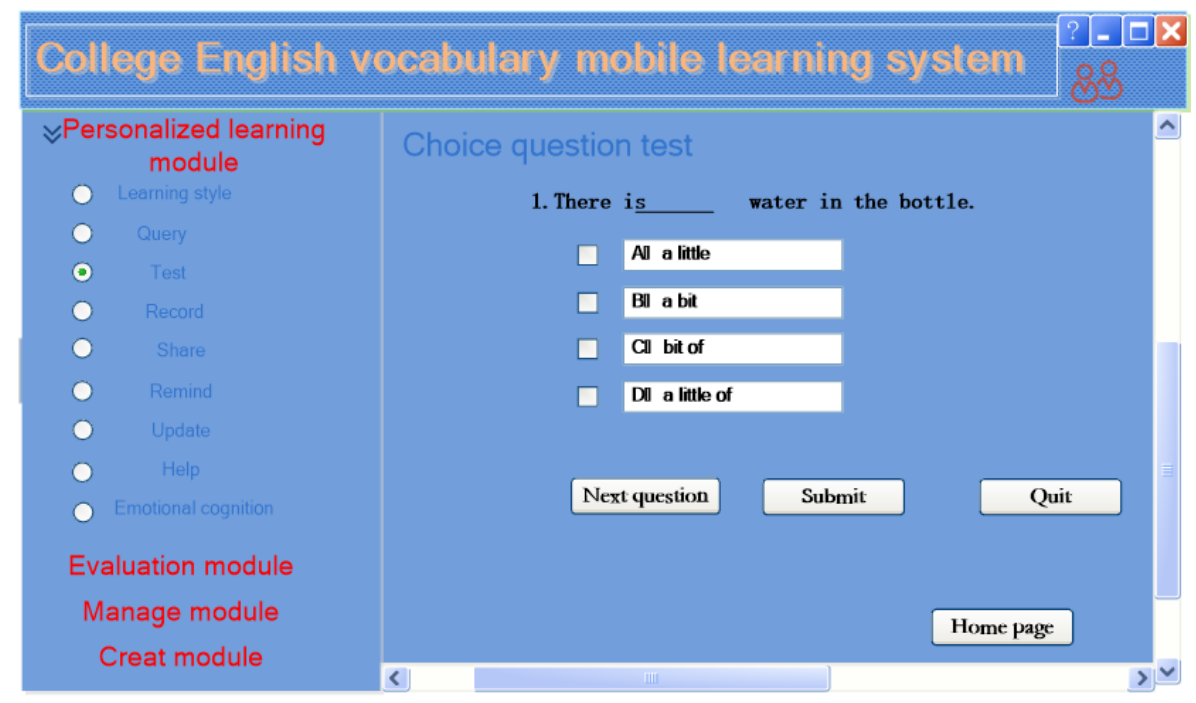

Fig. 5. The test interface of personalized learning module 


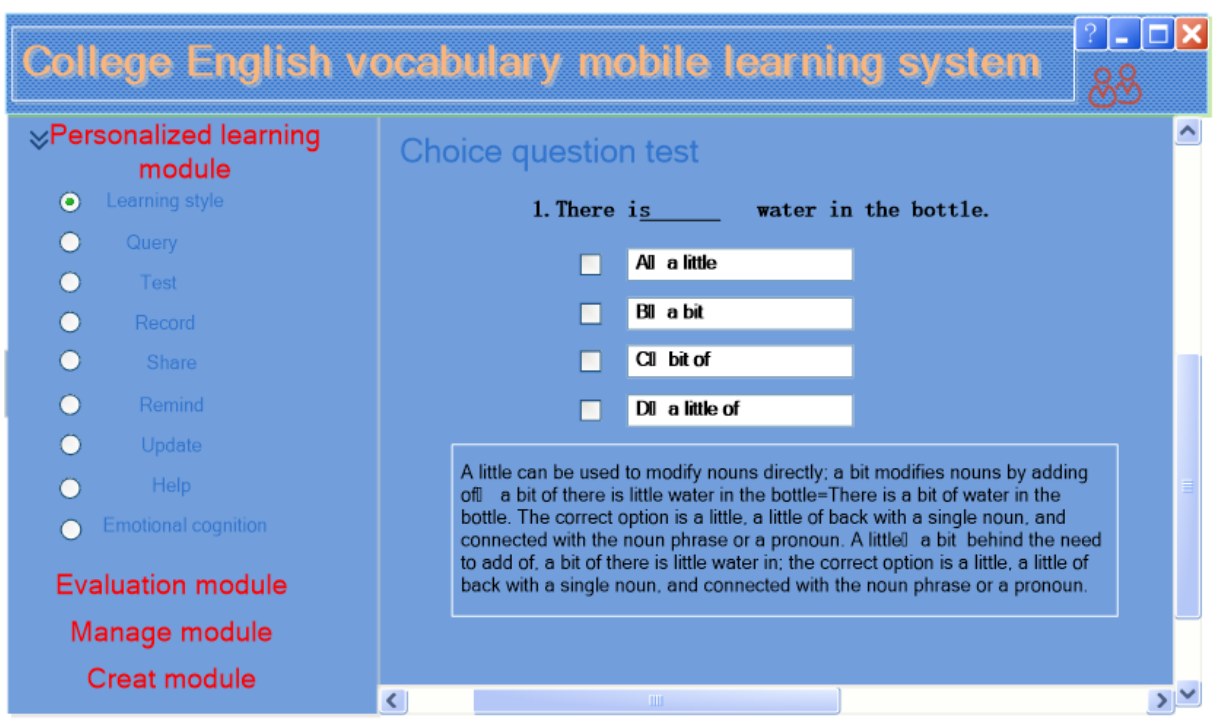

Fig. 6. The learning style interface of personalized learning module

\section{Conclusions}

This article aims to establish a mobile learning system for college students. Firstly, the hot topics and background of mobile learning at home and abroad are studied, and the key technologies related to mobile learning elaborated. Then, a CAD theoretical model is proposed based on the combination of a variety of learning theories. As to the system's functional requirements and module requirements, experiential research and questionnaire survey are used for a survey on students, and the results are analyzed and summarized. The main contents and significance of this article are as follows.

1. A cognitive theoretical model of Conversation - Activity - Distribution (CAD) is proposed, which greatly enriches the theoretical content of mobile learning.

2. The system's functional requirements and module requirements are investigated in combination with reality, which can better meet the scholars' demand.

3. The college English vocabulary mobile learning system is designed and realized, and the convenience of mobile learning and its ability to meet the individual needs are proven. Mobile learning model should be popularized and applied.

\section{$7 \quad$ References}

[1] Park, S., Foreman, W. A. (2009). Internationalization of higher education in Korea: the role of English-medium instruction, Environmental Conservation, 4(2), 1-19.

[2] Luján Tubio, M., Valencia, L., Clavijo, S. B., Allen, N. (2008). Using English in colombian companies: a pathway to internationalization and increased productivity, Current Microbiology, 57(4), 306-311. 
[3] Maiorov, V. (2006). Approximation by neural networks and learning theory, Journal of Complexity, 22(1), 102-117. https://doi.org/10.1016/j.jco.2005.09.001

[4] Song, M. J., Ahrndt, S. (1996). Incorporating instant messaging and video conferencing in content-based college English classrooms: a joint-course model, Neurosurgery, 39(3), 537547.

[5] Hurtado, J. E. (2004). An examination of methods for approximating implicit limit state functions from the viewpoint of statistical learning theory, Structural Safety, 26(3), 271293. https://doi.org/10.1016/j.strusafe.2003.05.002

[6] Ooi, K. B., Tan, W. H. (2016). Mobile technology acceptance model: an investigation using mobile users to explore smartphone credit card, Expert Systems with Applications, 59, 33-46. https://doi.org/10.1016/j.eswa.2016.04.015

[7] Zhao, D. (2017). Design on College English Vocabulary Learning System based on Mobile Phone APP. International Conference on Materials Science, Machinery and Energy Engineering, 24, 34-39.

[8] Anaraki, F. B. (2008). Development of a flash-based mobile learning system for English as second language, British Journal of Pharmacology, 75(2), 409-414.

[9] Wyckoff, M. M., Alsafran, S., \& Graygo, J. M. (2012). Use of mobile learning module improves skills in chest tube insertion - journal of surgical research, Journal of Surgical Research, 177(1), 21-26. https://doi.org/10.1016/j.jss.2012.03.022

[10] Hong, S. J., Thong, J. Y. L., \& Tam, K. Y. (2006). Understanding continued information technology usage behavior: a comparison of three models in the context of mobile internet, Decision Support Systems, 42(3), 1819-1834. https://doi.org/10.1016/j.dss.2006.03.009

[11] Engeström, Y. (2000). Activity theory as a framework for analyzing and redesigning work, Ergonomics, 43(7), 960. https://doi.org/10.1080/001401300409143

[12] Wickens, C. D. (2008). Situation awareness: review of mica endsley's 1995 articles on situation awareness theory and measurement, Human Factors, 50(3), 397. https://doi.org/10.1518/001872008X288420

[13] Abuzainab, N., Saad, W., Hong, C. S., \& Poor, H. V. (2017). Cognitive hierarchy theory for distributed resource allocation in the internet of things, IEEE Transactions on Wireless Communications, (99), 1-1. https://doi.org/10.1109/TWC.2017.2743077

[14] Che, P. C., Lin, H. Y., Jang, H. C., Lien, Y. N., Tsai, T. C. (2009). A study of English mobile learning applications at national chengchi university, International Journal of Distance Education Technologies, 7(4), 38-60. https://doi.org/10.4018/jdet.2009062403

[15] Shih, R. C., Cheng, Y. M., Kuo, S. H., Lou, S. J. (2016). The development and implementation of u-msg for college students' English learning, International Journal of Distance Education Technologies, 14(2), 17-29. https://doi.org/10.4018/IJDET.2016040102

\section{Authors}

Xin Zhang, Master of Arts for Applied Linguistics for English Language Education, Lecturer, Graduated from the University of Southampton in 2004, Worked in Dalian Polytechnic University. Her research interests include English language education and applied linguistics.

Jingxuan Bi, Master of Arts, lecturer, Graduated from Dalian Maritime University in 2005, Worked in Dalian Medical University, Her research interests include mobile learning, English teaching, applied linguistics

Article submitted 20 February 2018. Final acceptance 25 March 2018. Final version published as submitted by the authors. 